\title{
Mycotoxin management through transformations - A review
}

\author{
Archana Negi*, Roopali Sharma, Nandani Shukla, Sourav Kumar Modak, Manjari Negi \\ and Bhupesh Chandra Kabdwal
}

Department of Plant Pathology, College of Agriculture, G.B. Pant University of Agriculture and Technology, Pantnagar- 263145, U.S. Nagar, (Uttarakhand), INDIA

*Corresponding author. E-mail: archanagbpuat.patho@gmail.com

Received: September 30, 2016; Revised received: May 23, 2017; Accepted: October 10, 2017

Abstract: Mycotoxins are toxic secondary metabolic products of various fungi, mainly belonging to the genera Fusarium (Trichothecenes, Zearalenone), Aspergillus (Aflatoxin) and Penicillium (Ochratoxin) and can be found in almost $25 \%$ of the world's agricultural commodities. These compounds are toxic to humans, animals and plants and therefore, efforts should be made to avoid mycotoxin contamination in food and feed. It has been estimated that at least 300 of these fungal metabolites are potentially toxic to animals and humans. In India $50 \%$ losses of agricultural commodities are due to postharvest losses. A number of physical and chemical approaches have already been taken to reduce the effect of mycotoxins, but due to certain limitations of physical and chemical strategies prompted search for other solutions to the mycotoxin hazards. Thus, there is an increasing public pressure for a safer and ecofriendly alternative to control these organisms. Consequently, a new approach is applied for managing mycotoxins through transformations that offer specific, efficient and eco-friendly way for detoxification of mycotoxins. This review aims to brings about the up-to-date management strategies mainly through transformation (genetic and bio) to prevent or reduce post harvest damages to the crops caused by storage fungi and the contamination of food and feed by mycotoxins. It will make aware of the new technologies or management methods for mycotoxins through transformation. The transformation methods may become the technology of choice, as they offer a specific, irreversible, efficient and environment friendly way of detoxification that leaves neither toxic residues nor any undesirable byproducts.

Keywords: Aspergillus flavus, Mycotoxin, Ochratoxin, Trichothecenes, Zearalenone

\section{INTRODUCTION}

Mycotoxins are a group of highly toxic secondary metabolites of the fungi mainly belonging to genera Fusarium, Aspergillus and Penicillium, produced under certain favourable environmental conditions,such as temperature, humidity, $\mathrm{pH}$, atmospheric gasses, photoperiod light intensity and water availability. The temperaturerange usually reported for fungal growth is broad $\left(10-35^{\circ} \mathrm{C}\right)$, with a few species capableof growth below or above this range. In general, the optimum temperature for mycotoxin production is below the optimum for growth.Moisture requirements of foodborne moulds are relatively low; most species grow at a $0.85 \mathrm{aw}$ (water activity) or less, although yeasts generally require a higher water activity. $0.60 a \mathrm{w}$ is considered the limit for cell growth, but spores of Aspergillus and Penicillium for example, are able to survive at lower $a \mathrm{w}$ for several years (Carlile and Watkinson, 1996). The acid/alkaline requirement for growth of all yeasts and moulds is quite broad, ranging from $\mathrm{pH} 3$ to above $\mathrm{pH} 8$, with optimum around $\mathrm{pH} \mathrm{5}$, if nutrient requirements are satisfied. In general, Aspergillus species are more tolerant to alkaline $\mathrm{pH}$ while Penicillium species appear to be more tolerant to acidic $\mathrm{pH}$ (Wheeler et al., 1991).

According to ICAR (2012) 50\% losses of agricultural commodities is due to post harvest losses in India. Among which maximum post harvest losses is for fruits $(18 \%)$, for vegetables $(12 \%)$, for oil seed $(10 \%)$ and for pulses and cereals it is $6 \%$. It has been estimated that at least 300 of these fungal metabolites are potentially toxic to animals and human. Human food can be contaminated with mycotoxins at various stages in the food chain (Bennett and Klich, 2003). Factors contributing to the presence or production of mycotoxins in foods or feeds include storage, environmental, and ecological conditions. Often times most factors are beyond human control (Hussein and Brasel, 2001). A wide range of commodities can be contaminated with mycotoxins both pre- and post-harvest (CAST, 2003). The wide range of effects and losses due to mycotoxins make their management and prevention of contamination in food products necessary. A number of physical and chemical approaches have already been taken to reduce the effect of mycotoxins, but due to certain limitations of physical and chemical strategies prompted a search for other solutions to the mycotoxin haz- 
ards. Consequently, a new approach is applied for managing mycotoxins through transformations by using genetic engineering and biotechnological techniques (genetic transformation) and; microbes and enzymatic detoxification (bio-transformation) that offer specific, efficient and eco-friendly way for detoxification of mycotoxins that leaves neither toxic residue nor any undesirable by-product.

According to FAO $25 \%$ of the world's crops are affected by mycotoxins with annual loss of 1 billion metric tons of food and food products. In India 50\% losses of agricultural commodities are due to post harvest losses.

Government of India, Ministry of food processing industries (2016): As a quarter of agricultural products loss is due to mycotoxin contamination and with the knowledge that mycotoxins can have serious effects on humans and animals has led many countries to establish regulations relating to mycotoxin in food to protect the consumer from harmful effects of these compounds. First time the limit is set for aflatoxin in late 1960's but by the end of 2003 more than 100 countries had developed specific limits for mycotoxins in food and feed. According to FAO (2013)25\% of the world's crops are affected by mycotoxins with annual loss of 1 billion metric tons of food and food products. In United States losses associated with deoxynivalenol were estimated at $\$ 655$ million/year, with the majority of the losses in wheat (APS, 2013).Losses due to aflatoxins in three Asian countries (Indonesia, Philippines, and Thailand) were estimated at $\$ 900$ million annually (APS, 2013).Diversity in the toxic effects and the synergetic properties of mycotoxins has made it mandatory to consider them as "risk factor"to humans and in livestock health (Yiannikouris and Jonany2002; D'iazLlano and Smith 2006). Concerns over health effects of mycotoxins are being seriously considered worldwide. Mycotoxicosis is the general term given for the disease that is caused by mycotoxins (Nelson and others 1993).

Implications of mycotoxins: Mycotoxins produce adverse effects on agricultural commodities, humans and animals and also may have role in pathogenicity.

Contaminate agricultural commodities (crop plants, fruits, seed and grains): the may cause qualitative as well as quantitative loss.

Impact on humans and animals: mycotoxin infected agricultural commodities causes mycotoxicosis when consumed by animals and humans. A well known example of mycotoxicosis is ergotism that occurs due to consumption of mycotoxin (ergopeptine and clavine)

Table 1. The Post-harvest loseses is as under.

\begin{tabular}{ll}
\hline Crops & Cumulative losses (\%) \\
\hline Cereals & $4.65-5.99$ \\
Pulses & $6.36-8.41$ \\
Oil seeds & $3.08-9.96$ \\
Fruits and Vegetables & $4.58-15.88$ \\
\hline
\end{tabular}

contaminated rye.

Mycotoxin may be phytotoxic: They may have potential role in pathogenesis. Aflatoxin produced from Aspergillus flavus and A. parasiticus, pathogenic on a variety of plant species are reported to be phytotoxin and may have potential role in pathogenesis. Trichothecens (deoxynivalenol) are produced by destructive pathogen Fusarium that attack wide range of plant species. There is acute phytotoxicity of tricothecens in plant tissues.

According to Joint Expert Committee on Food Additives (JECFA) which is a scientific advisory body of FAO and WHO (that provide mechanism for assessing the toxicity of food additive), mycotoxins which are currently considered most significant are- Aflatoxin, Ochratoxin A, Zearalenone, Fumonisins, Trichothecenes(deoxynivalenol), Patulin.

Aflatoxin: It is the first mycotoxin to be reported in 1960 when death of thousands of turkey occurs due to consumption of contaminated peanuts, meals (Turky'X' disease). These are produced by Aspergillus species especially $A$. flavus and A. parasiticus. Frequently affected cereals, oil-seeds, various spices.

There are 24 types of aflatoxin but only four are found in food stuff i.e. aflatoxin B1, B2, G1, G2. Aflatoxin may also be found in the milk of animals that feed on aflatoxin contaminated food (aflatoxin M1 and M2). Toxicity of aflatoxin is due to epoxide ring present at 8 -9 carbon position in its chemical structure. These are genotoxic \& carcinogenic and can cause liver cancer in humans, chronic hepatitis and jaundis.

The biosynthetic pathway of aflatoxin includes; a Type -I polyketide synthase (PKS) and a pair of specialized fatty acid synthase subunits (FAS-1 and FAS-2) to synthesize norsolorinic acid. Then a series of around 15 redox reactions mediated by cytochromes $\mathrm{P} 450$ (isoenzyme system) occurs that leads to the production of aflatoxins. The biosynthetic pathway progresses as under:

Norsolorinic acid (NOR) $\rightarrow$ Averantin $(\mathrm{AVN}) \rightarrow 5^{\prime}-$ hydroxyaverantin $($ HAVN) $\rightarrow$ Oxoaverantin $($ OAVN) $\rightarrow$ Averufanin $\quad($ AVNN) $\rightarrow$ Averufin $\quad(\mathrm{AVF})$ $\rightarrow$ Versiconal hemiacetal acetat (VHA) $\rightarrow$ Versiconal $(\mathrm{VAL}) \rightarrow$ Versicolorin B (VERB) $\rightarrow$ Versicolorin A (VERA) $\rightarrow$ Demethylsterigmatocystin $\rightarrow$ Dihydrodemethylsterigmatocystin (DMST) $\rightarrow$ Sterigmacystin (ST) $\rightarrow$ Dihydrosteig (DHDMST) (DHST) $\rightarrow O$-Methylsterigmatocystin $\rightarrow$ Dihydro-O-methylsterigmatocystin (OMST) (DHOMST) $\rightarrow$ Aflatoxin $\quad \mathrm{B}_{1} \quad\left(\mathrm{AFB}_{1}\right) \rightarrow$ Aflatoxin $\quad \mathrm{B}_{2} \quad\left(\mathrm{AFB}_{2}\right)$ $\rightarrow$ Aflatoxin $\mathrm{G}_{1}\left(\mathrm{AFG}_{1}\right) \rightarrow$ Aflatoxin $\mathrm{G}_{2}\left(\mathrm{AFG}_{2}\right)(\mathrm{Yu}$, 2012)

Ochratoxin: These are particularly produced by $A s$ pergillus sp. (A. Ochraceous, A. parasiticus, A. niger) and Penicillium sp.. Ochratoxin are of three typesOchratoxin A, B, and C. Among these ochratoxin A is most important; it contaminates commodities like, ce- 
Table 2 . Some human diseases in which mycotoxins have been implicated.

\begin{tabular}{lll}
\hline Disease & Mycotoxin source & Fungus \\
\hline Akakabio-byo & Wheat, barley, oat, rice & Fusarium spp. \\
Alimentary toxic aleukia & Cereal grains (toxic bread) & Fusarium spp. \\
Balkan nephropathy & Cereal grains & Penicillium spp. \\
Cardiac beriberi & Rice & Aspergillus spp., Penicillium spp. \\
Celery harvester's disease & Celery (Pink rot) & Sclerotinia \\
Ergotism & Rye, cereal grains & Claviceps purpurea \\
Hepatocarcinoma & Cereal grains, peanuts & Aspergillus flavus, A. parasiticus \\
Kwashiorkor & Cereal grains & Aspergillus flavus, A. parasiticus \\
Neural tube defects & Maize & Fusarium verticillioides, F. proliferatum \\
Oesophageal tumors & Corn & Fusarium verticillioides, F. proliferatum \\
Onyalai & Millet & Phoma sorghina \\
Reye's syndrome & Cereal grains (grain dust) & Aspergillus \\
Stachybotryotoxicosis & Cereal grains (grain dust) & Stachybotrys atra \\
\hline
\end{tabular}

Source Bryden, W. L. (2007)

reals, coffee, grape juices and spices. Ochratoxin cause kidney damage in swine and are carcinogenic to humans. Ochtratoxin-A (OTA) is a very important mycotoxin for food security and human health, but very little is known about the genetic background of OTA biosynthesis as compared to the other important mycotoxins such as aflatoxins, fumonisins and zearalenones (37). L-Phenylalanine was recognized as a precursor of OTA synthesis (Wang et al., 2016). OTA biosynthetic pathway had been proposed as a scheme by Huff and Hamilton.They hypothesized that mellein (dihydroisocoumarin, a phenolic compound produced by Aspergillus ochraceus), catalyzed by Polyketide Synthase PKS, was oxidized to OT $\beta$ and then transformedto OT $\alpha$ by a halogenase/chloroperoxidase. Subsequently, OT $\alpha$ was esterified to ochratoxin C via linkwith the ethyl ester, and finally biosynthesized to OTA by a deesterification reaction (Huff et al., 1979).

Zearalenone and Zearalenol: These are produced by Fusarium sp. particularly F. Gramminearum mainly in maize. It is estrogenic mycotoxinoccurring in Fusarium-infected cereals,causing hormonal effects (Michlmayr et al., 2017). It is also associated with the ear or stalk rot of corn, but its role in pathogenesis is not yet defined.

Trichothecenes: These are one of the most recognised groups of mycotoxins, these includes over 60 compounds but the most effective are deoxynivalenol (DON), T-2, HT-2, nivalenol, diacetoxyscirpenol (DAS). These are associated with commodities likewheat, maize, rice, oats, rye, barley, mainly produced by Fusarium sp. The toxicity is due to epoxy group present at 12-13 carbon position. They cause acute and chronic mycotoxicosis in farm animals and humans. These mycotoxins may also have role in pathogenesis and causes ear rot in corn and scab \& head blight in wheat, barley, oats and rye.

Biosynthesis of Fusarium trichothecenes begins with the cyclization of farnesyl pyrophosphate (primary metabolic intermediate) to form trichodiene. The enzyme terpene cyclase trichodiene synthase (Tri5) catalyzes this reaction (Hohn et al., 1986).Trichodiene undergoes a series of oxygenations catalyzed by a cytochrome $\mathrm{P} 450$ monooxygenase. The four oxygens were added at C-2, C-3, C-11, and the C-12, C-13epoxide to form theintermediate isotrichotriol. Isotrichotriol then, undergoes a non-enzymatic isomerization and cyclization to form isotrichodermol $(=3 \alpha-$ hydroxy EPT) (Mc Cormick et al., 1990).During this process, the oxygen at the $\mathrm{C}-2$ position becomes the

Table 3. Important mycotoxins produced by different fungi.

\begin{tabular}{ll}
\hline Fungus & Mycotoxin \\
\hline Aspergillus flavus and A. parasiticus & Aflatoxin \\
A.Fumigatus & Fumagillin \\
A.versicolor, A. nidulans, and A. rugulosus & Sterigmatocystin \\
A. Ochraceus, Penicillium viridicatum & Ochratoxin \\
P.verrucosum & Ochratoxin \\
P. citrinum, P. viridicatum & Citrinin \\
P. patulum, P. expansum & Patulin \\
P. puberulum, P. purpurogenum & Penicillic acid \\
Fusarium sp. & Trichothecenes and Zearalenone \\
F. tricinctum, F. graminearum, F. equiseti, F. sporotrichioides, F. & T-2 toxin, HT-2 and Diacetoxyscirpenol(DAS) \\
poae and F. lateritium & \\
F. graminearum, F. tricintum & Zearalenone and Zearalenol \\
F. graminearum & Deoxynivalenol(DON) or Vomitoxin \\
F. moniliforme & Fumonisins \\
Claviceps purpurea & Ergot alkaloides(ergopeptine and clavine) \\
\hline
\end{tabular}


Table 4. Different physical and chemical methods for mycotoxin management.

\begin{tabular}{lll}
\hline S. N. & Physical approaches & Chemical approaches \\
\hline $\mathbf{1 .}$ & Washing & Treatment of contaminated feed with ammonia \\
$\mathbf{2 .}$ & Polishing & $\begin{array}{l}\text { Treatment of agricultural commodities by oxidising and reducing agents, } \\
\text { acids, bases, salts and chlorinating substances have been tested for their abil- } \\
\text { ity to degrade mycotoxins }\end{array}$ \\
& Mechanical sorting and saperation & \\
4. & Density segregaton & \\
$\mathbf{5 .}$ & Floatation & \\
$\mathbf{6 .}$ & Autoclaving & \\
7. & Roasting and microwave heating & \\
$\mathbf{8 .}$ & UV irradiation & \\
$\mathbf{9 .}$ & Ultrasound treatment & \\
10. & Solvent extraction &
\end{tabular}

Source : Heidler ( 2003)

Table 5. Some of microorganisms that are to detoxify mycotoxin.

\begin{tabular}{ll}
\hline Microorganism & Mycotoxin \\
\hline Agrobacterium sp. and Eubacterium sp. & Deoxynivalenol \\
Saccharomyces cerevisiae and Paeciliomyces sp. & Patulin \\
Flavobacterium aurantiacum and Aspergillus flavus & Aflatoxin \\
Exophiala pinifera, Rhinocladiella atrovirens and Bacterium ATCC 55552 & Fumonisins \\
Gliocladium roseum NRRL 1859, Rhodococcus etythropolis & Zearalenone \\
Curtobacterium sp., Butyvibrio fibrisolvens and Selenomonas ruminantium & T-2 toxin \\
\hline
\end{tabular}

pyran ringoxygen and the hydroxyl group at $\mathrm{C}-11$ is lost. More complex Type A trichothecenes are built bymodifying isotrichodermol through a series of paired hydroxylation $(-\mathrm{OH})$ and acetylation or acylation $(-\mathrm{OR})$ steps. Isotrichodermol $(\mathrm{C}-3-\mathrm{OH})$ is converted to isotrichodermin ( $\mathrm{C}-3-\mathrm{OR})$ by an acetyltransferase. A second hydroxyl group is added to C-15. A third hydroxyl group is added at C-4. A fourth hydrox$\mathrm{yl}$ group is added to $\mathrm{C}-8$ followed by the addition of an isovaleryl moiety. Finally, the acetyl group is removed from the $\mathrm{C}-3$ position by an esterase to produce trichothecene (Mc Cormick et al., 2011)

Fumonisins: There are various groups of fumonisins. Most important are A1, A2, B1, B2. These are produced from Fusarium sp. (F. Moniliformae, F. Verticilloides, F.proliferate). Corn is the mostly affected commodities. These are carcinogenic to humans \& may causes oesophageal cancer. It causes liver \& kidney toxicity in animals. These may also play role in pathogenesis. It's been found that more than $95 \%$ concentration of fumonisins produced by Giberella fusikuroi increases virulence on maize seedling. The Fumonisin (FB1) backbone is structurally very similar to sphinganine, a fatty acid-derived intermediate in sphingolipid metabolism (Merrill et al., 1997). However, it is not clear whether fumonisins are derived from a fatty acid or polyketide because both types of compounds are derived from acetate.

Management of mycotoxins: Due to harmful effects of mycotoxins to animals, plant and humans it is necessary to manage mycotoxin contamination in agricultural commodities. A number of physical and chemical approaches have already been taken to reduce the ef- fect of mycotoxins. The efficiency of these techniques depends on the level of contamination and the distribution of mycotoxins in produce. Therefore, the results obtained are uncertain and often connected with high product losses. Moreover, some of these treatments are relatively costly and may remove or destroy essential nutrients in food and feed. The limitation of physical and chemical strategies prompted a search for other solutions to the mycotoxin hazard. Consequently, a new approach is applied for managing mycotoxins is through transformations by using genetic engineering and biotechnological techniques (genetic transformation) and; microbes and enzymatic detoxification (bio-transformation) that offer specific, efficient and eco-friendly way for detoxification of mycotoxins that leaves neither toxic residue nor any undesirable byproduct.

However, the efficiency of these techniques depends on the level of contamination and the distribution of mycotoxins throughout the grain. Subsequently the results obtained are uncertain and often connected with high product losses. Moreover, some of these physical treatments are relatively costly and may remove or destroy essential nutrients in feed. Chemical methods require not only suitable reaction facilities but also additional treatments (drying, cleaning) that can make them time consuming and expensive. Only a limited number of these are effective without diminishing the feed's nutritional value or palatability (Heidler, 2003)

Genetic transformation: There are three strategies through which genetic transformation can be done

Reducing infection by the pathogen: In this strategy a noval gene is inserted that expresses antifungal pro- 
teins or secondary metabolites such as - hydroxamic acid and phenolics. It induce existing plant defence pathway in warding off infection.

Inserting genes capable of degrading the toxin: Fumonisin is greatly detoxified by this strategy. Gene for Fumonisin esterase is been isolated \& cloned from Exophiala spinifera which is capable of metabolizing Fumonisin. Then these genes are exposed in maize plants by using biotechnological tools. Then expression of these genes on Fumonisin level kernels is being evaluated.

Reducing mycotoxin accumulation by interfering with the biosynthetic pathway: This strategy involves engineering plants to produce proteins or compounds that interfere with mycotoxin biosynthesis, or altering plant genome, so it fails to produce signalling compounds involved in mycotoxin biosynthesis, for example- in case of aflatoxin, $\alpha$-amylase helps in its synthesis and $\alpha$-amylase inhibitor proteins can inhibit aflatoxin synthesis identified from legume Lablabpurpureus. So genetically altered maize plant can be formed having $\alpha$-amylase inhibitor.

Different gene transfer method: There are various gene transfer methods through which desirable gene is transmitted to plant. There is direct DNA gene transfer method and vector mediated gene transfer method. Among direct method there are some physical and chemical methods, while vector mediated transfer method is through Agrobacterium mediated method.

Physical methods

Electroporation: In this method there is use of electric impulse to produce pores in plasma membrane, thereby allowing macromolecules into the cells for the uptake of DNA.Here plant material is incubated in buffer containing target DNA then subjected to high voltage electric impulse.

Particle bombardment: It uses accelerated microprojectiles to deliver DNA into intact tissues \& cells. For this purpose gene guns are been devised that literally fires DNA into target cells. The DNA which is to be transformed into the cells is coated with tungsten or gold particle. The coated particles are loaded into the firing chamber of the gene guns which is accelerated to high speed towards the target cell. The projectile penetrates the plant cell walls and membrane.

Microinjection: In this method needles or micropipettes are used for injecting DNA. This method is tried in mainly cereals.

Silicon carbide fibre mediated transformation: DNA coated with silicon carbide fibres are vortexd with plant material. DNA adhered with the fibres enters the cells \& get integrated with host genome.

\section{Chemical methods}

Polythene glycol mediated method: In this method isolated protoplast of the plant cell is kept in a solution containing $40 \%$ polyethylene glycol (PEG), calcium nitrate $/ \mathrm{CaCl}$ and mannitol/Sucrose also present as os- motic buffering agent into the solution. Then carrier DNA is added into the solution. After exposure of DNA to protoplast PEG is allowed to remove.

Calcium phosphate Co-precipitation mediated method: In this method isolated DNA is mixed with solution of Calcium chloride \& Potassium phosphate buffer under a condition which allow to form Calcium phosphate precipitate having DNA. Plant cell are incubated with precipitated DNA either in solution or in culture dish. Fraction of cell will take up the DNA precipitate by endocytosis.

Vector mediated gene transfer method: Agrobacterium tumefaciens has been widely applied as a vector for plant transformation. Agrobacterium tumefaciens is a plant pathogenic bacteria causing crown gall disease. This bacterium is having Ti plasmid that contains tumour genes that alter host plant metabolism in favour of pathogens. This bacterium is considered as natures little plant transformer and has been exploited to mediate the introduction of gene of interest into the target plant cells with the help of T-DNA from Ti plasmid.

Ti plasmid contains three components- virulence gene region, T-DNA region and opine catabolism region. TDNA region having tumour inducing genes or genes for biosynthesis of auxin and cytokinin and opine. Virulence genes region having genes that are responsible for transfer of T-DNA. It codes for protein involved in T-DNA transfer. At least nine vir genes operons has been identified these are virA, virG, virB, virC1, virD1, virD2, virD4, virE1, virE2. Opine catabolism region codes for protein involved in the uptake $\&$ metabolism of opines.

In this method firstly the gene of interest is inserted into the T-DNA region. Wounded plant cell produce phenolic defence compound which can triggers the expression of the bacterium's vir genes. It encoded for vir protein that process the T-DNA region from $\mathrm{Ti}$ plasmid and form $\mathrm{T}$-strand. After the bacterium attack plant cell, the T-strand and several vir proteins are transformed to the plant through a transport channel. Inside the plant cell, the vir proteins interact with the $\mathrm{T}$ -strand, forming a T-complex. This T-complex targets the nucleus, allowing the T-DNA to integrate into the plant genome and results in the expression of the encoded genes.

Previously many studies have shown that genetic transformations can be used to manage mycotoxins by using specific gene responsible to detoxify a specific mycotoxin. For instance, detoxification of deoxynivalenol (DON) and Zearalenone (ZEN) were achieved by expression of acetyltransferase gene (Tri101) and lactonohydrolase gene (Zh101) respectively (Okubura et al., 2002; Takahashi et al., 2004). Thus with more knowledge in molecular plant pathology and biotechnology, problem of mycotoxin in agricultural commodities can be solved by inserting genes that can detoxify the mycotoxins. 
(B) Bio-transformation: Bio-transformation was demonstrated first time by Binder et al. (2000). Bacterial strain having detoxification activity for Trichothecenes by selective cleavage at 12-13 epoxy groups was isolated from bovine rumen. Mycotoxin deactivating feed additive based on this bacterial isolate was developed and was named as BBSH. Several microbes have been identified having mycotoxin degradation activities. Detoxification of Ochratoxin A (OTA) and Patulin (PAT) by Lactic Acid bacteria Fuchs et al. (2007). Alberts et al. (2009) studied the enzymatic degradation of aflatoxin B1 by rot fungi in different liquid medium. They isolated different white fungi at different media and tested for there laccase activity and degradation in all medium. It was found that Pleurotus ostreatus and Peniophora sp. shows maximum laccase activity and thus maximum degradation of mycotoxin. Islam et al., 2012 studied and describes a novel bacterial culture isolated from agricultural soil that was capable of transforming (deoxynivalenol) DON to deepoxy DON under aerobic and anaerobic conditions at moderate temperatures.Twenty-three yeast species, including four Trichomonascus species and 19 anamorphic species were tested for their ability to convert the trichothecene T-2 toxin to less-toxic products( $\mathrm{Mc}$ Cormick, et al., 2012). The Human Fecal Microbiota Metabolizes Deoxynivalenol and Deoxynivalenol-3Glucoside (Gratz, et al., 2013).

\section{Conclusion}

Mycotoxins have huge significant in post harvest loss and managing it is not an easy task. Transformation is a new approach to manage mycotoxin affected commodities by using biotechnological \& genetic engineering techniques and enzymatic or microbial degradation that offers a specific, efficient, environmental friendly way of detoxification of mycotoxin and leaves neither toxic residue nor any undesirable by product. But since this approach is in its infant stage so further work into enzymology, genetic modification, residual toxicity and identification of gene for detoxification are required to harness the potential of these microbes that is used as feed additive or from which gene is extracted.

Future prospects: With more knowledge in molecular plant pathology and use of biotechnology techniques GM crops may offer a possible contribution to manage mycotoxin.

To determine whether the ability of the product (microorganism) to biologically degrade mycotoxin under in vivo or storage condition.

In case of bio-transformation, the responsible detoxification system (genes involved in process) remains to be identified.

The environmental safety of genetically engineered (GE) crops and foods, just as with those created by classical breeding and mutation and grown conven- tionally or organically, must be evaluated on a case-bycase basis to perform meaningful risk assessments

\section{REFERENCES}

Alberts, J.F., Gelderblom, W.C.A., Botha, A. and Zyl, W.H.V. (2009). Degradation of aflatoxin B1 by fungal laccase enzymes. International Journal of Food Microbiology. 135:47-52

APS (2013). American Phytopathological Society, USA

Bennett, J.W. and Klich, M. (2003). Mycotoxins. Clin. Microbiol. Rev. 16: 497-516

Binder, E. M., D. Heidler, G. Schatzmayr, N. Thimm, E. Fuchs, M. Schuh, R. Krska and J. Binder. (2000). Microbial detoxification of mycotoxins in animal feed. Proceedings of the 10th International IUPAC Symposium on Mycotoxins and Phycotoxins, Brazil

Bryden, W. L. (2007). Mycotoxins in the food chain: human health implications. Asian Pac J Clin Nutri. 16 (Suppl 1): $95-101$

Carlile, M.J. and Watkinson, S.C. (1996). The fungi. Academic Press, Harcourt Brace and Company Publishers, London, UK

CAST (2003). Mycotoxins: Risks in Plant, Animal and Human Systems. Report No.139. Council for Agricultural Science and Technology, Ames, Iowa, USA

D'1az-Llano G and Smith T.K. (2006). Effects of feeding grains naturally contaminated with Fusarium mycotoxins with and without a polymeric glucomannan mycotoxin adsorbent on reproductive performance and serum chemistry of pregnant gilts. J Anim Sci ., 84:2361-66

FAO (2013). Food and Agriculture Organization

Fuchs, S., Sontag, G., Stidl, R., Ehrlich, V., Kundi, M. and Knasmu, S. (2008). Detoxification of patulin and ochratoxin A, two abundant mycotoxins, by lactic acid bacteria. Food and Chemical Toxicology. 46:1398-1407

Gratz, S.W., Duncan, G., Richardson, A.J. (2013). The Human Fecal Microbiota Metabolizes Deoxynivalenol and Deoxynivalenol-3-Glucoside and May Be Responsible for Urinary Deepoxy-Deoxynivalenol. Applied and Environmental Microbiology, 79(6): 1821-1825

Heidler, D. and Schatzmayr, G. (2003). A new approach to managing mycotoxins. World Poultry - Reed. 19(2)

Hohn, T.M., Middlesworth, F.V. (1986). Purification and characterization of the sesquiterpene cyclase trichodiene synthetase from Fusarium sporotrichioides. Arch. Biochem. Biophys., 251: 756-761

Huff, W. and Hamilton, P. (1979). Mycotoxins-their biosynthesis in fungi: Ochratoxins-metabolites of combined pathways. J. Food Prot. 42:815-820

Hussein, H.S. and Brasel, J.M. (2001). Toxicity, metabolism, and impact of mycotoxins on humans and animals. Toxicology.167:101-134

Islam, R., Zhou, T., Young, J. C., Goodwin, P. H. and Pauls, K.P. (2012). Aerobic and anaerobic de-epoxydation of mycotoxin deoxynivalenol by bacteria originating from agricultural soil. World J Microbiol Biotechnol.28:7-13

Mc Cormick, S.P., Stanley, A. M., Stover, N.A. and Alexander, N.J. (2011). Trichothecenes: From Simple to Complex Mycotoxins. Toxins. 3: 802-814

Mc Cormick, S.P., Taylor, S.L., Plattner, R.D., Beremand, M.N. (1990). Bioconversion of possible T-2 toxin precursors by a mutant strain of Fusarium sporotrichioides. Appl. Environ. Microbiol.56:702-706 
Mc Cormick, S.P., Price, N.P.J. and Kurtzman, C.P. (2012). Glucosylation and Other Biotransformations of T-2 Toxin by Yeasts of the Trichomonascus Clade. Appl. Environ. Microbiol. 78 (24):8694-8702

Merrill, A. H., Schmelz, E.-M., Dillehay, D. L., Spiegel, S., Shayman, J. A., Schroeder, J. J., Riley, R. T., Voss, K. A., and Wang, E. (1997). Sphingolipids - The enigmatic lipid class: Biochemistry, physiology, and pathophysiology. Toxicol. Appl. Pharmacol. 142: 208-225

Michlmayr, H., Varga, E., Lupi, F., Malachova, A., Hametner, C., Berthiller, F. and Adam G. (2017).Synthesis of Mono- and Di-Glucosides of Zearalenone and _ $\alpha-/ \beta$ Zearalenol by Recombinant Barley Glucosyltransferase HvUGT14077.Toxins. 9:58

Nelson, P. E., Desjardins, A. E. and Plattner, R. D. (1993). Fumonisins, mycotoxins produced by Fusarium species: biology, chemistry and significance. Annu. Rev. Phytopathol.31:233-252

Okubura, P.A. and Blechl, A.E(2002).Engineering deoxynivalenol metabolism in wheat through the expression of a fungal trichothecene acetyltransferase gene. Theor.
Appl. Genet. 106:74-83

Takahashi-Ando, N., Ohsato, S., Shibata, T., Hamamoto, H., Yamaguchi, I. and Kimura, M (2004). Metabolism of zearalenone by genetically modified organisms expressing the detoxification gene from Clonostachys rosea. Applied and Environmental Microbiology. 70(6):32393245

Wang Y., Wang L., Liu F., Wang Q., Selvaraj J N., Xing F., Zhao Y. and Liu, Y. (2016). Ochratoxin A Producing Fungi, Biosynthetic Pathway and Regulatory Mechanisms Toxins . 8:83

Wheeler, K.A., Hurdman, B.F. and Pitt, J.I. (1991) Influence of $\mathrm{pH}$ on the growth of sometoxigenic species of Aspergillus, Penicillium and Fusarium. International Journal of Food Microbiology. 12:141-150

Yiannikouris, A. and Jonany, J. (2002). Mycotoxins in feeds and their fate in animals: a review. Anim Res51:81-9

Yu, J. (2012). Current Understanding on Aflatoxin Biosynthesis and Future Perspective in Reducing Aflatoxin Contamination. Toxins. 1024-1057 